\title{
Lipomatosis gástrica: reporte de un caso
}

\section{Gastric lipomatosis: a case report}

Manuelita Ramos-Calderón, MD, ${ }^{*}$ Jaime Solano-Mariño, MD, ${ }^{2}$ Rocío del Pilar López-Panqueva, MD. ${ }^{3}$

Médica rural de investigación, Departamento de Patología y Laboratorios, Hospital Universitario Fundación Santa Fe de Bogotá. Bogotá D. C., Colombia. Código ORCID: https://orcid. org/0000-0001-8776-1803

2 Cirujano gastroenterólogo. Servicio de gastroenterología, Hospital Universitario Fundación Santa Fe de Bogotá. Bogotá D. C. Colombia. Código ORCID: https://orcid. org/0000-0001-9650-1501

3 Patóloga, Departamento de Patología y Laboratorios, Hospital Universitario Fundación Santa Fe de Bogotá. Bogotá D. C., Colombia. Código ORCID: https://orcid.org/0000-00017277-7482

*Correspondencia: Manuelita Ramos-Calderón, MD manule19@gmail.com

Fecha recibido: $\quad 30 / 04 / 19$

Fecha aceptado: 17/06/19

\section{Resumen}

La lipomatosis gástrica es una enfermedad infrecuente caracterizada por múltiples lesiones tumorales benignas (lipomas), que acorde a su tamaño pueden producir una variedad de síntomas. Por lo general, la enfermedad es documentada incidentalmente en estudios imagenológicos que se realizan para estudiar otras enfermedades y los hallazgos patológicos contribuyen a tener una certeza en el diagnóstico de esta patología. Por el momento, no hay un tratamiento definido para las masas pequeñas y asintomáticas, mientras que para las masas grandes $(>3-4 \mathrm{~cm})$ o sintomáticas se sugiere la resección quirúrgica de las lesiones.

\section{Palabras clave}

Neoplasias gástricas, endoscopía gastrointestinal, lipoma, tomografía, histología.

\section{Abstract}

Gastric lipomatosis is a rare disease characterized by multiple lipomas, benign tumors which can produce a variety of symptoms according to their size. In general, the disease is incidentally documented in imaging studies done to study other diseases. Pathological findings can contribute to the certainty of diagnosis. At the moment, there is no definite treatment for small, asymptomatic masses, but surgical resection is suggested for masses that are larger than 3 or $4 \mathrm{~cm}$ and for those that are symptomatic.

Keywords

Gastric neoplasms, gastrointestinal endoscopy, lipoma, tomography, histology.

\section{INTRODUCCIÓN}

Los lipomas gastrointestinales son tumores mesenquimales poco frecuentes y constituyen menos del $3 \%$ de los tumores gástricos. La lipomatosis gástrica es una condición muy infrecuente, solo se encuentran algunos reportes de caso en la literatura. Se caracteriza por la presencia de múltiples lipomas gástricos o por la infiltración difusa de la submucosa o subserosa por el tejido adiposo maduro. No existen reportes de transformación maligna. Se presenta el caso de una mujer de 65 años, asintomática, en quien se observaron múltiples masas subepiteliales gástricas. El estudio image- nológico e histopatológico confirmó el diagnóstico de lipomatosis gástrica.

\section{CASO CLÍNICO}

Una mujer de 65 años con un diagnóstico previo de pólipos gigantes gástricos acudió al servicio de gastroenterología para realizar el seguimiento de estas lesiones mediante un ultrasonido endoscópico, en ese momento la paciente se encontraba asintomática. La paciente refirió antecedente de dislipidemia en manejo farmacológico y negó antecedentes familiares de lipomas. En el examen físico, el único hallazgo 
relevante era la presencia de 2 lipomas en las extremidades inferiores, el de mayor tamaño estaba localizado en la cara anterior del muslo derecho, a nivel del trocánter mayor, con un diámetro aproximado de $4 \mathrm{~cm}$; el de menor tamaño se encontraba en la cara posterior del muslo izquierdo, por encima de la fosa poplítea y tenía un diámetro de $2 \mathrm{~cm}$.

En el examen gastroscópico, se documentaron seis lesiones elevadas subepiteliales, polipoides, sésiles, cubiertas por mucosa sana, localizadas en el antro y sobre la curvatura mayor del estómago; la lesión de mayor tamaño medía 2,7 x $1,7 \mathrm{~cm}$. Estos hallazgos plantearon diagnósticos diferenciales, por lo que se decidió realizar estudios complementarios para definir el manejo médico o quirúrgico.

Se realizó ecoendoscopia, en la que se identificó la lesión de mayor tamaño, con un diámetro de $22 \mathrm{~mm}$, con origen en la submucosa, hiperecoica, homogénea y sin estructuras vasculares en su interior; se tomó una biopsia por aspiración con aguja fina, en la que solo se lograron observar extendidos hemorrágicos sin células epiteliales ni mesenquimales; el material no fue diagnóstico. Posteriormente, se realizó una tomografía axial computarizada (TAC) de abdomen que refirió el hallazgo de múltiples lesiones gástricas, localizadas sobre la curvatura mayor nodulares y redondeadas, con densidad grasa (-100 UH) que correspondían a múltiples lipomas, sin evidencia de ganglios perigástricos ni compromiso abdominal o en la pelvis, y con un hallazgo adicional de enfermedad diverticular del colon izquierdo sin signos de inflamación; no se encontraron otras lesiones (Figura 1).

Por los hallazgos descritos, se realizó la resección endoscópica de la lesión de mayor tamaño para confirmar la etio- logía de las lesiones (Figura 2). Al estudio histopatológico se observó una lesión tumoral mesenquimal, benigna localizada en la submucosa, constituida por una proliferación de adipocitos maduros, con escasos septos fibrosos, sin presencia de atipia celular, mitosis ni necrosis (Figura 3). Con estos hallazgos clínicos, el tratamiento de elección fue conservador y se continuó con controles rutinarios de las lesiones gástricas por endoscopia. La paciente hasta la fecha ha evolucionado satisfactoriamente.

\section{DISCUSIÓN}

La lipomatosis gástrica es una enfermedad poco frecuente, solo se han reportado 12 casos en la literatura; a diferencia de los lipomas gástricos únicos, que representan el $3 \%$ de todos los tumores benignos gástricos. Por lo general, la lipomatosis gástrica tiende a ser asintomática y hasta el momento no se han reportado casos con transformación maligna (1-3). Esta se define por la presencia de múltiples lipomas gástricos o por la infiltración difusa de tejido adiposo en la capa de la submucosa o subserosa del estómago $(1,4)$; no obstante, no está definido el número mínimo de lipomas que deben presentarse para llamarlo lipomatosis gástrica; normalmente más de 4 lipomas se considera lipomatosis gástrica (5). Los lipomas son de consistencia blanda, pequeños en tamaño, con bordes bien definidos, de forma redondeada u ovalada y sésiles, pero se han reportado casos de lipomas pedunculados (6). Su localización de preferencia es la submucosa, la cual se presenta en el $90 \%-95 \%$ de los casos, con un $5 \%-10 \%$ de los casos que surgen en la subserosa. Anatómicamente, se encuentran
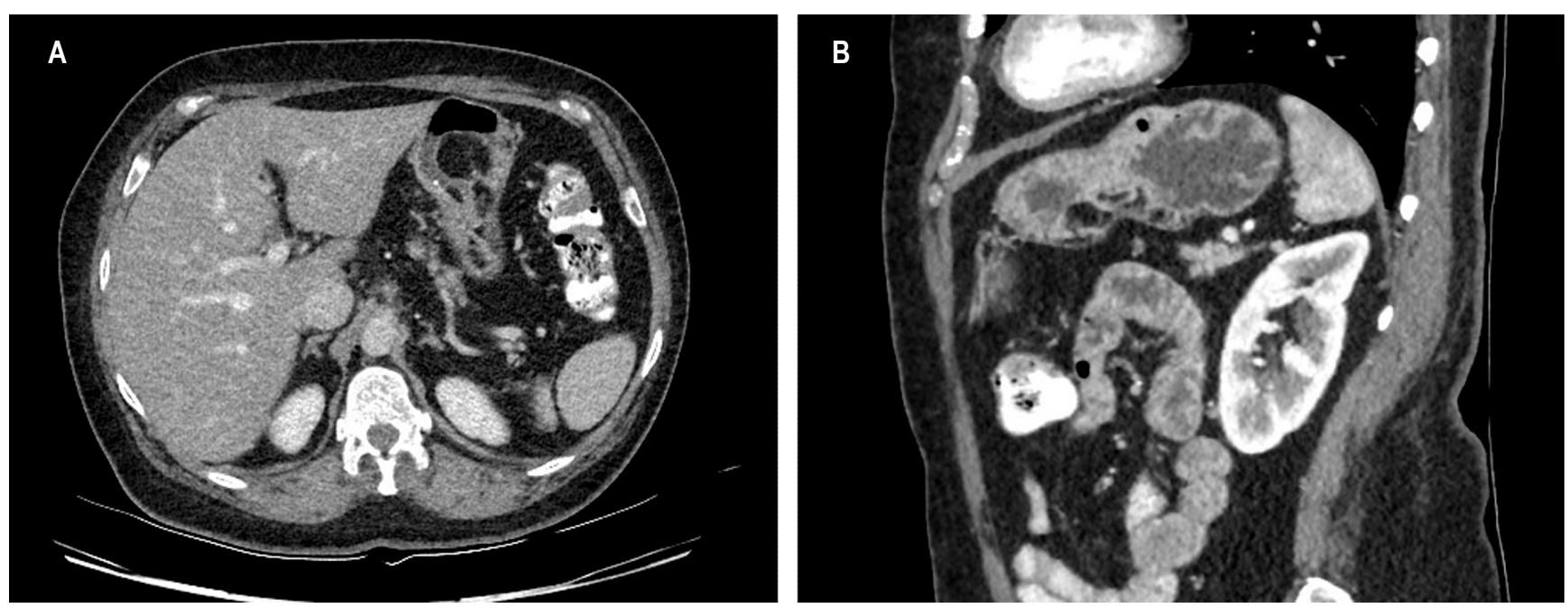

Figura 1. TAC de abdomen. A lo largo de la curvatura mayor del estómago se observan múltiples lesiones nodulares y redondas con densidad grasa (-100 UH) de localización submucosa transmural. A. Vista axial. B. Vista sagital. 

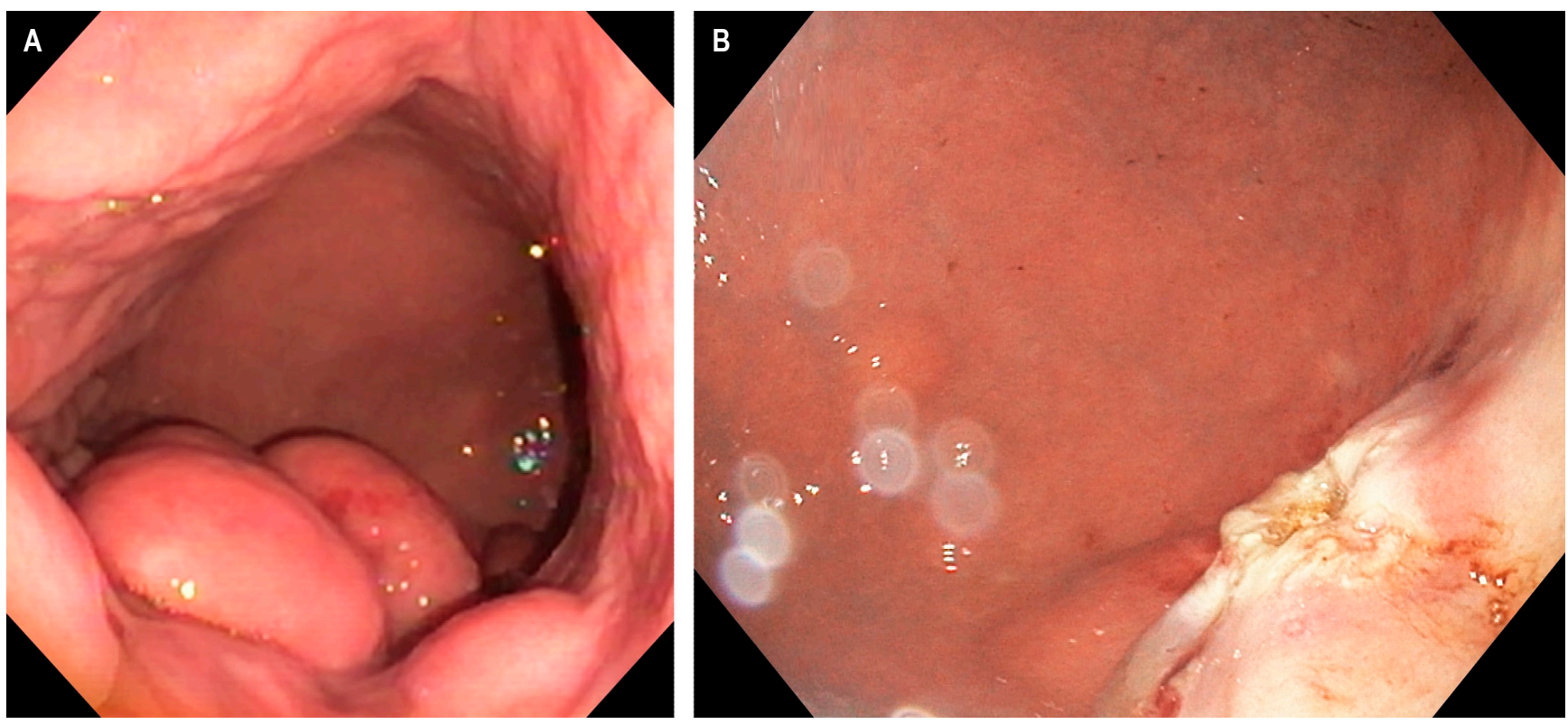

Figura 2. Endoscopia. A. Se observan múltiples lesiones elevadas submucosas cubiertas por mucosa sana en la curvatura mayor del estómago. B. Resección de la lesión de mayor tamaño.

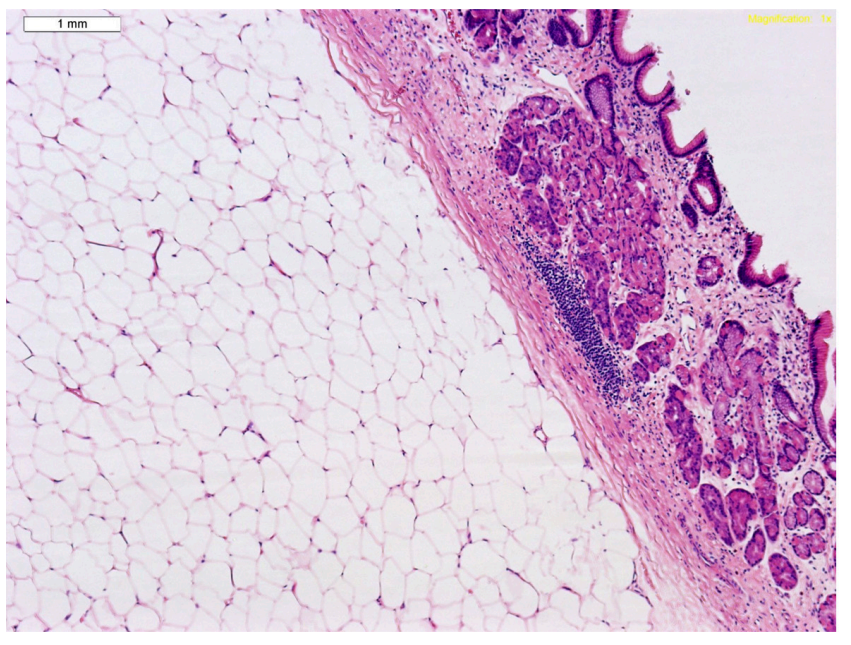

Figura 3. H\&E X 20. Hallazgos histológicos muestran lesión tumoral en la submucosa gástrica constituída por la proliferación de tejido adiposo maduro, sin atipia celular, mitosis, ni necrosis.

principalmente en el antro (75\%), sin reportes actuales de aparición en el cardias o píloro $(3,7)$. Histológicamente, consisten en una proliferación de tejido adiposo maduro, con septos fibrovasculares delgados, rodeados por una cápsula fibrosa $(5,7)$.
Por el momento, no se conoce exactamente la etiología de estas lesiones, aunque existen hipótesis de su posible origen como el desplazamiento embrionario del tejido adiposo, una predisposición genética, alteraciones en el metabolismo de la grasa, depósitos de grasa posterior a quimioterapia o irritación crónica $(1,7)$. Adicionalmente, en un número de casos se ha asociado la lipomatosis gástrica con la lipomatosis familiar múltiple, una enfermedad genética benigna rara, con una prevalencia estimada de 0,002\% en la población general (8-11). Usualmente, los pacientes presentan múltiples lipomas indoloros en el tejido adiposo subcutáneo de las extremidades y del tronco que empiezan a aparecer a partir de la tercera y cuarta décadas de la vida $(11,12)$. Adicionalmente, algunos estudios refieren la posibilidad de que los pacientes presenten dislipidemia concomitantemente; sin embargo, el perfil lipídico puede aparecer sin alteraciones $(13,14)$. Por lo general, esta enfermedad se hereda de manera autosómica dominante, aunque se han reportado casos de herencia recesiva $(8,9$, 13). Hasta el momento no se ha identificado una mutación específica para la lipomatosis familiar múltiple, pero en ciertas ocasiones se han realizado estudios genéticos y de cariotipo que han reportado asociaciones con mutaciones en el ácido desoxirribonucleico (ADN) mitocondrial, en el gen PALB2 (socio y localizador del gen BRCA2) y en el gen HMGA2 (12, 14-17). 
Habitualmente la lipomatosis gástrica no genera síntomas y su hallazgo está dado incidentalmente en autopsias o en estudios clínicos para otras entidades (3). Cuando los pacientes presentan sintomatología, esta se relaciona principalmente con el tamaño de las lesiones, siendo más frecuentes los síntomas con masas mayores de 2 a $4 \mathrm{~cm}$ (3, 18). La presentación clínica más frecuente es la hemorragia gastrointestinal (50\%), que puede estar asociada con dolor epigástrico, náuseas y vómitos. Estos síntomas obstructivos generalmente aparecen al inicio de la enfermedad y son más frecuentes cuando los lipomas se encuentran en el antro o son lipomas pedunculados que generan intususcepción del estómago $(1-3,6)$. En cuanto a la hemorragia gástrica, esta se debe a la ulceración de la mucosa por los lipomas que se produce por produce por estasis venosa, cuando los lipomas tienen una gran masa o es secundaria a la inflamación, que hace más susceptible la mucosa a la isquemia $(2,19)$. El sangrado gastrointestinal puede ser agudo o crónico, por lo que se deben ampliar los estudios clínicos, dado que los pacientes pueden estar cursando con anemia y deficiencia de hierro.

Actualmente, la TAC de abdomen es la herramienta imagenológica de elección ya que los lipomas tienen un patrón específico dado por una estructura homogénea, una forma regular, ausencia de crecimiento infiltrativo y una densidad grasa característica de -80 a -120 unidades Hounsfield (HU). Cuando en la TAC se observan hebras basilares de tejido blando en un lipoma uniforme sugiere la presencia de una úlcera en la lesión $(4,18,20)$. La resonancia magnética también es útil para identificar los lipomas por su alta sensibilidad para detectar el tejido adiposo y es indicación en niños y pacientes alérgicos al medio de contraste (3).

En la endoscopia se pueden observar tres signos que sugieren la presencia de un lipoma: el signo de la tienda, que es la disposición de los pliegues gástricos hacia la lesión; el signo de la almohada, que hace referencia a la sensación táctil al oprimir una esponja; y la protrusión de grasa a través de la masa secundaria a la toma de múltiples biopsias $(2,3)$. Una limitación de las biopsias tomadas durante la endoscopia radica en que no se obtiene una apropiada representación del tejido graso ubicado en la submucosa $(7,21)$. Otra herramienta de diagnóstico es la ultrasonografía endoscópica, ya que permite evaluar la capa donde se origina el tumor y se pueden realizar biopsias de la submucosa $(2,4,22)$.

En cuanto al tratamiento de elección, aún no existe un acuerdo para establecer una guía o tratamiento estándar, principalmente porque las lesiones son asintomáticas y por la baja incidencia que presenta esta condición. Algunos autores sugieren que el plan de tratamiento debe basarse en el tamaño de la lesión y de acuerdo con la sintomatología que presente el paciente; para masas pequeñas y asintomáticas se sugieren controles periódicos de las lesiones, mientras que la resección quirúrgica está recomendada en masas grandes (> $3-4 \mathrm{~cm}$ ) y en masas sintomáticas, ya que estas pueden generar complicaciones posteriores. En cuanto a masas pequeñas y asintomáticas aún no existe un consenso en cuanto a si se debe realizar cirugía profiláctica o no $(1,2,7)$.

\section{CONCLUSIÓN}

La lipomatosis gástrica es una condición muy inusual. Se presentó el caso de una mujer asintomática, sin antecedentes familiares de lipomas, a quien se le encontraron en la endoscopia múltiples masas subepiteliales gástricas y la presencia de lipomas en las extremidades. La caracterización por imágenes y la resección endoscópica confirmaron el diagnóstico. Debe tenerse en cuenta este diagnóstico diferencial ante la presencia de masas subepiteliales gástricas.

\section{REFERENCIAS}

1. Jeong IH, Maeng YH. Gastric lipomatosis. J Gastric Cancer. 2010;10(4):254-8.

https://doi.org/10.5230/jgc.2010.10.4.254

2. Alberti D, Grazioli L, Orizio P, Matricardi L, Dughi

S, Gheza L, Falchetti D, Caccia G. Asymptomatic giant gastric lipoma: What to do? Am J Gastroenterol. 1999;94(12):3634-7.

https://doi.org/10.1111/j.1572-0241.1999.01391.x

3. López-Zamudio J, Leonher-Ruezga KL, Ramírez-González LR, Jiménez GR, González-Ojeda A, Fuentes-Orozco C. Lipoma gástrico pediculado. Reporte de caso. Cir y Cir.
2015;83(3):222-6. https://doi.org/10.1016/j.circir.2015.05.005

4. Aoyama S, Ami K, Fukuda A, Imai K, Chong J-M, Ando M. Gastric lipomatosis treated by total gastrectomy: a case report. Surg Case Reports. 2017;3(1):126. https://doi.org/10.1186/s40792-017-0404-1

5. Liu X, Wilcox CM, Nodit L, Lazenby AJ. Multiple gastrointestinal stromal tumors and lipomatosis. Arch Pathol Lab Med. 2008;132(11):1825-9.

6. Peabody JW, Ziskind J. Lipomatosis of the stomach A case report and a review of the literature. Ann Surg. 
1953;138(5):784-90.

https://doi.org/10.1097/00000658-195311000-00017

7. Juan F, Gallego JI, Gómez C, Guirau MD. Lipoma gástrico: un nuevo caso y revisión bibliográfica. Radiologia. 2001;43(6):300-2. https://doi.org/10.1016/S0033-8338(01)76980-9

8. Arabadzhieva E, Yonkov A, Bonev S, Bulanov D, Taneva I, Ivanova $\mathrm{V}$, Dimitrova $\mathrm{V}$. A rare combination between familial multiple lipomatosis and extragastrointestinal stromal tumor. Int J Surg Case Rep. 2015; 14:117-20. https://doi.org/10.1016/j.ijscr.2015.07.027

9. D’Ettorre M, Gniuli D, Guidone C, Bracaglia R, Tambasco D, Mingrone G. Insulin sensitivity in familial multiple lipomatosis. Eur Rev Med Pharmacol Sci. 2013;17(16):2254-6.

10. Leffell DJ, Braverman IM. Familial multiple lipomatosis: Report of a case and a review of the literature. J Am Acad Dermatol. 1986;15(2):275-9. https://doi.org/10.1016/S0190-9622(86)70166-7

11. Djuric-Stefanovic A, Ebrahimi K, Sisevic J, Saranovic D. Gastroduodenal lipomatosis in familial multiple lipomatosis. Med Princ Pract. 2017;26(2):189-91. https://doi.org/10.1159/000454714

12. Lee CH, Spence RAJ, Upadhyaya M, Morrison PJ. Familial multiple lipomatosis with clear autosomal dominant inheritance and onset in early adolescence. BMJ Case Rep. 2011;2010-2. https://doi.org/10.1136/bcr.10.2010.3395

13. Keskin D, Ezirmik N, Celik H. Familial multiple lipomatosis. IMAJ. 2002;4:1121-3.

14. Sayar I, Demirtas L, Gurbuzel M, Isik A, Peker K, Gulhan B. Familial multiple lipomas coexisting with celiac disease: A case report. J Med Case Rep. 2014;8(1):1-4. https://doi.org/10.1186/1752-1947-8-309

15. Reddy N, Malipatil B, Kumar S. A rare case of familial multiple subcutaneous lipomatosis with novel PALB2 mutation and increased predilection to cancers. Hematol Oncol Stem
Cell Ther. 2016;9(4):154-6.

https://doi.org/10.1016/j.hemonc.2016.01.001

16. Prontera P, Stangoni G, Manes I, Mencarelli A, Donti E. Encephalocraniocutaneous lipomatosis (ECCL) in a patient with history of familial multiple lipomatosis (FML). Am J Med Genet Part A. 2009; 149A(3):543-5. https://doi.org/10.1002/ajmg.a.32692

17. Vaughan CJ, Weremowicz S, Goldstein MM, Casey M, Hart M, Hahn RT, Devereux RB, Girardi L, Schoen FJ, Fletcher JA, Morton CC, Basson CT. A t $(2 ; 19)(\mathrm{p} 13 ; \mathrm{p} 13.2)$ in a giant invasive cardiac lipoma from a patient with multiple lipomatosis. Genes Chromosom Cancer. 2000;28(2):133-7. https://doi.org/10.1002/(SICI)10982264(200006)28:2<133::AID-GCC1>3.0.CO;2-K

18. Taylor J, Stewart T, Dodds J. Gastrointestinal lipomas: A radiologic and pathologic review. Am J Roentgenol. 1990;155(December):1205-10. https://doi.org/10.2214/ajr.155.6.2122666

19. Skinner MS, Broadaway RK, Grossman P, Seckinger D. Multiple gastric lipomas. Dig Dis Sci. 1983;28(12):1147-9. https://doi.org/10.1007/BF01295816

20. Ferrozzi F, Tognini G, Bova D, Pavone P. Lipomatous tumors of the stomach: CT findings and differential diagnosis. J Comput Assist Tomogr. 2000;24(6):854-8. https://doi.org/10.1097/00004728-200011000-00006

21. Ventura L, Leocata P, Guadagni S, Ventura T. Multiple gastric lipomas: Report of an asymptomatic case found at autopsy. Pathol Int. 1997;47(8):575-7. https://doi.org/10.1111/j.1440-1827.1997.tb04543.x

22. Dias de Castro F, Magalhães J, Monteiro S, Leite S, Cotter J. The Role of Endoscopic Ultrasound in the Diagnostic Assessment of Subepithelial Lesions of the Upper Gastrointestinal Tract. GE Port J Gastroenterol. 2016;23(6):287-92. https://doi.org/10.1016/j.jpge.2016.05.001 